\title{
Kikuchi-Fujimoto Disease Presenting as Brachial Plexus Neuritis: A Case Report
}

\author{
Rakesh Gupta $^{1}$ Akshat Kayal ${ }^{1}$ Vishwesh Rajawat ${ }^{2}$ \\ ${ }^{1}$ Department of Neurosurgery SAIMS Medical College and P.G. \\ Institute, Indore, Madhya Pradesh, India \\ 2 Department of Pathology, SAIMS Medical College and P.G. Institute, \\ Indore, Madhya Pradesh, India \\ Indian J Neurosurg 2016;5:110-113.
}

Address for correspondence Akshat Kayal, MS, Department of Neurosurgery, SAIMS Medical College and P. G. Institute, IndoreUjjain State Highway, Indore, Madhya Pradesh 452018, India (e-mail: drakshatkayal@gmail.com).

\author{
Abstract \\ Keywords \\ - Kikuchi-Fujimoto \\ disease \\ - brachial plexopathy \\ - histiocytic necrotizing \\ lymphadenitis \\ - lymphadenopathy
}

Kikuchi-Fujimoto disease is a rare, idiopathic, self-limiting necrotizing lymphadenitis of unknown etiology. It usually affects young Asian women. Clinically, it closely resembles with tuberculosis or lymphomas. This pathology has occasionally been associated with various cardiac, renal, pulmonary, and other systemic complications. The association of the disease in context to other neurological pathologies is even more sporadic. We report a case of Kikuchi-Fujimoto disease in a 56-year-old woman associated with the rare presentation as brachial plexus neuritis. The diagnosis was established histopathologically upon surgical resection of lesion. We discuss the peculiar features of presentation, pathological elements, surgical management, and postoperative outcome with special emphasis on complications of this clinical entity.

\section{Introduction}

Persistent cervical and axillary lymphadenopathy is a common occurrence encountered in clinics across Asian countries. The commonest presentation in young population is secondary to infections of which extrapulmonary tuberculosis tops the list. Occasionally, one encounters neoplastic lesions such as lymphomas. ${ }^{1}$ In adults, these aberrant swelling warrant exclusion of metastatic malignant disease.

Kikuchi-Fujimoto disease (KFD) or subacute histiocytic necrotizing lymphadenitis is a benign, rare, self-limiting disease, which usually presents itself with cervical or axillary lymphadenopathy associated with fever. ${ }^{2}$ Initially described in Japan, KFD was first reported in 1972 independently by Kikuchi and Fujimoto et al. It has a higher prevalence among Asiatic individuals, often young adults younger than 30 years with a female preponderance (female-to-male ratio, 4:1) ${ }^{3}$

We describe the case of a middle aged Indian woman who presented with cervical and axillary lymphadenopathy due to KFD and its peculiar presentation as brachial plexus neuritis.

received

April 6, 2015

accepted

December 18, 2015

published online

July 14, 2016

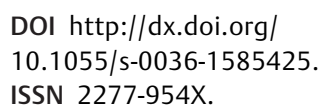

\section{Case Report}

A 56-year-old woman presented with 2.5 months' history of fever, associated with swelling, pain, and redness on the left side of neck and scapular region. She received several courses of antibiotics without any benefit. Gradually, her pain started radiating to left shoulder and was accompanied with paresthesia of left upper limb. Progressive worsening of left hand grip was also noticed simultaneously. She denied sudden onset of weight loss, dysuria, cough, and respiratory distress. There was no previous history of tuberculosis or contact with a patient suffering from it, and she had no family history of leukemia, lymphoma, systemic lupus erythematosus (SLE), rheumatoid arthritis, or any other rheumatological disorders.

She had weakness in left elbow flexors, wrist flexors, finger abductors and adductors, and thumb flexors and extensors. There was mild hypoesthesia over left C5 to C7 dermatome. Regional examination revealed left tender supraclavicular and axillary lymphadenopathy. The largest node was palpable in the left supraclavicular region and was $\sim 3 \times 3 \mathrm{~cm}$ in size. In addition, the nodes were also palpable in the anterior, central, and apical group of the axillary
License terms

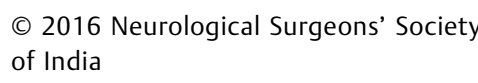
of India

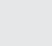 \\ .
}


lymph nodes. The cardiovascular, respiratory, and abdominal systems were clinically normal.

Her blood investigations were unremarkable without any evidence of leucopenia. Additionally, Mantoux test and polymerase chain reaction for mycoplasma were negative. The chest X-ray showed no abnormality of bony thoracic cage with bilaterally normal lung fields. An ultrasound of abdomen displayed an unremarkable study.

Magnetic resonance imaging (MRI) of cervical spine demonstrated a large lymph node measuring $\sim 5 \times 5 \mathrm{~cm}$ in the left supraclavicular region compressing over the upper brachial plexus in the short $\mathrm{T} 1$ inversion recovery (STIR) sequences (-Fig. 1). In addition, there were multiple lymph nodes along the cervical (level IV), superior mediastinum, left subclavian, and axillary vessels. Incidentally, the patient also had an annular disc bulge at C5-6 with right paracentral disc protrusion and right oncovertebral osteophytes causing mild narrowing of the right neural foramina and abutting the intraspinal nerve root.

Fine needle aspiration smears from the lesion exhibited mature lymphocytes, reactive lymphoid cells, and macrophages without evidence of granuloma or atypical cells. An excision biopsy of the largest supraclavicular lymph node was performed, upon which the left upper limb symptoms of the patient resolved completely.

The excised specimen macroscopically had solid greyish surface with large area of necrosis. The microscopic histopathology of sections showed features of necrotizing lymphadenopathy with characteristic stellate necrosis rimmed with histiocytes and immunoblasts and karyorrhectic debris without granulocytic infiltration. Multinucleate giant cells or atypical cells were absent. Staining for acid-fast bacilli was also negative (-Fig. 2). Diagnosis of KFD was made on the basis of histological findings.

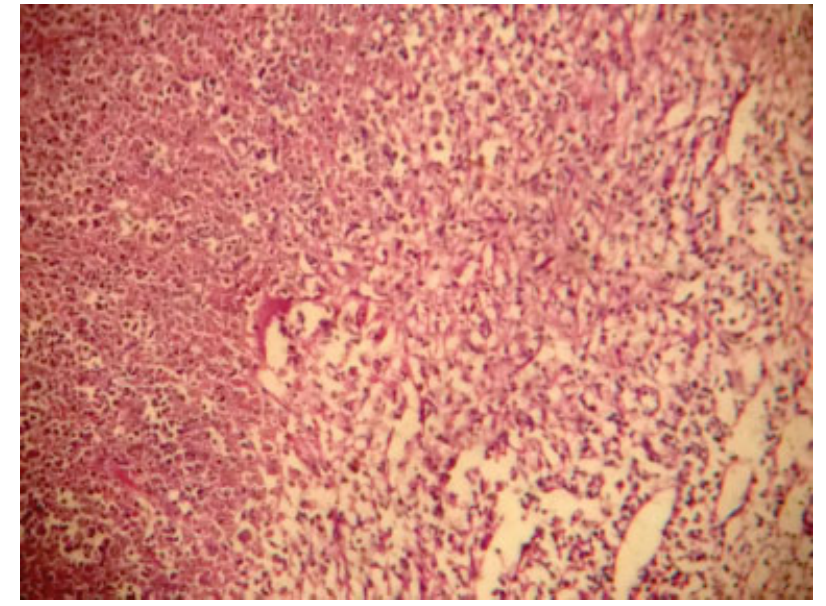

Fig. 2 Histopathology of lesion showing features of necrotizing lymphadenopathy with characteristic stellate necrosis rimmed with histiocytes and immunoblasts and karyorrhectic debris without granulocytic infiltration.

Following surgery, the patient was put on a regime of antibiotics, analgesics, and steroids for 4 weeks to which patient effectively responded with resolution of neurological deficits. A repeat scan after 4 months displayed significant resolution of lymphadenopathy and patient remains asymptomatic till date (-Fig. 3).

\section{Discussion}

KFD is an extremely rare disease that shows sporadic worldwide distribution, but has a higher prevalence amongst Japanese and other Asiatic individuals. ${ }^{3}$ Its occurrence in the Indian subcontinent is rare. Mostly,

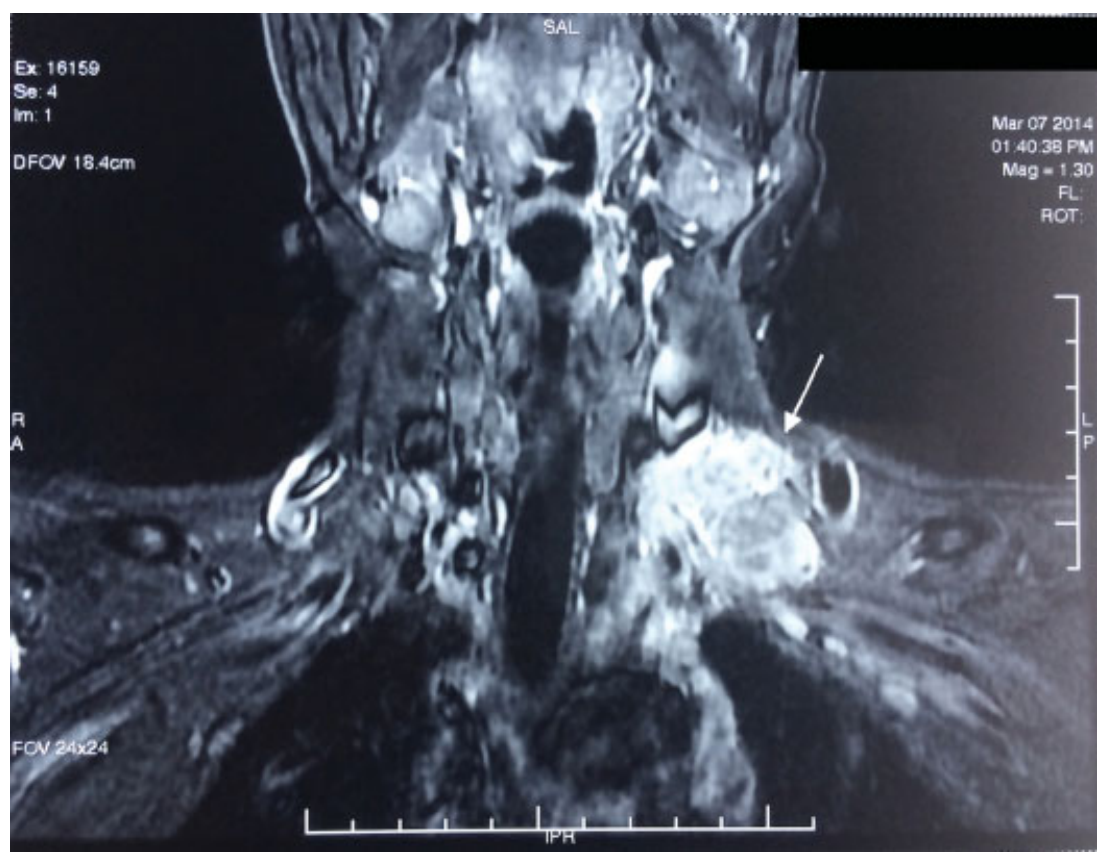

Fig. 1 Magnetic resonance imaging (short T1 inversion recovery) of cervical spine showing a large lymph node (arrow) in the left supraclavicular region compressing over the brachial plexus. 


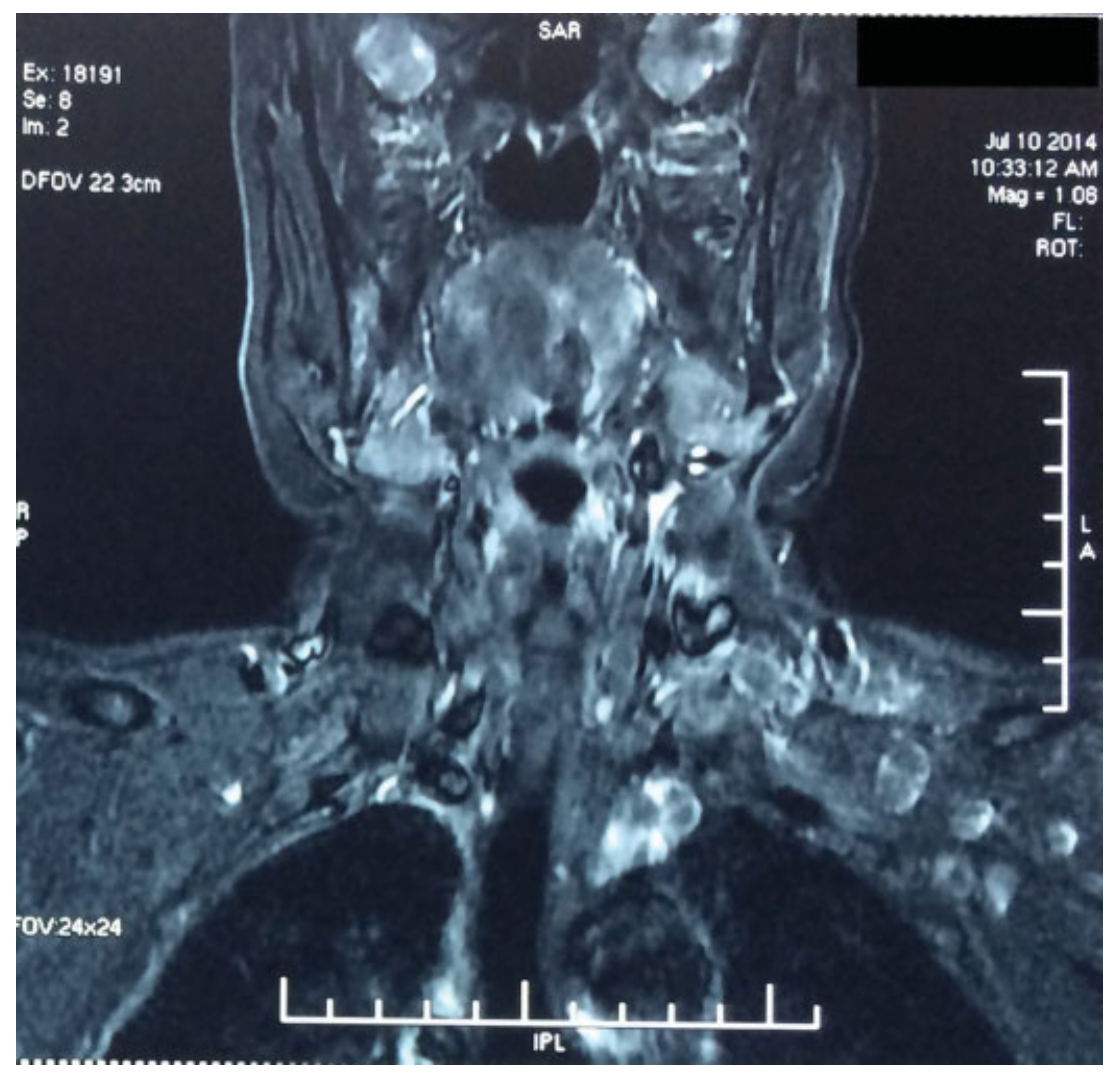

Fig. 3 Postoperative magnetic resonance imaging with reduction of lymphadenopathy.

sporadic case reports find a mention from India and surrounding region. The largest series is from Pakistan wherein Wahid et al documented 22 cases in the past decade. ${ }^{4}$ The disease has a definite trend to manifest itself in young adults with an inclination toward female preponderance. Mean age in various studies ranges from 25 to 30 years. Although patients have also presented later in life as noted in studies by Dorfman and Berry ${ }^{2}$ (mean age, 30; range, 11-75) and Kucukardali et $\mathrm{al}^{5}$ (mean age, 25; range, 1-64). Our patient presented with the disease at the age of 56 years.

Etiology of KFD is under speculation. Viral agent's role in pathogenesis of KFD remains controversial and not convincingly demonstrated. Epstein-Barr viruses, human immunodeficiency virus, human T-lymphotrophic virus 1 , herpes simplex virus, and parvovirus B19 have been suggested as causative agents. Bacterial agents such as Toxoplasma, Yersinia, Bartonella, and Brucella have also been implicated as causative agents. ${ }^{6}$ Alternatively, an autoimmune mechanism has been proposed as electron microscopic studies have identified tubular reticular structures in cytoplasm of stimulated lymphocytes and histiocytes in KFD patients. These are also been identified in cells of patients with SLE. ${ }^{7}$ None of the causative agents or mechanisms could be identified in our case.

The onset of KFD is acute or subacute, evolving over period of 2 to 3 weeks. As in our patient, cervical lymphadenopathy manifested as tender neck swellings and fever, usually of low grade that are the hallmarks of the disease. ${ }^{3,4}$ Patients, less commonly, may also have symptoms of arthralgia, skin rashes, weakness, and night sweats. Few reports of weight loss, diarrhea, anorexia, nausea, vomiting, chest, and abdominal pain are documented. Some patients may also have hepatosplenomegaly. ${ }^{6}$

Interestingly, KFD has been associated with various systemic complications, which occasionally are completely unrelated to the disease itself. Panuveitis, cardiac tamponade, isolated mediastinal lymphadenopathy, mesenteric lymphadenopathy, acute renal failure, hepatitis, hemophagocytic syndrome, interstitial lung disease, and pleural effusion have all been reported individually. In addition, rare central nervous system complications like meningitis and aseptic encephalitis have also found mention. Peripheral neuropathy has been reported as well. ${ }^{8}$ To the best of our knowledge, there is only a single case report in the literature wherein Sugiyama et al observed involvement of brachial plexus with right upper limb paresis in a 22-year-old woman. ${ }^{9}$ Brachial plexus neuritis was the unique feature of our case as well.

Differential diagnosis of KFD includes lymphoma, tubercular adenitis, lymphogranuloma venereum and Kawasaki disease. Contrast-enhanced computed tomography and MRI (T2 and STIR sequences) identify the enlarged lymph nodes distinctly and are good follow-up tools to observe disease remission as well.

Owing to the acute onset and prolonged but benign, selflimiting course of the disease, the treatment paradigm essentially encompasses achieving symptomatic relief for 
the patient. Analgesics-antipyretics and nonsteroidal antiinflammatory drugs are helpful in alleviating the symptoms of fever and lymph node tenderness. Studies also mention use of antibiotics and corticosteroids in severe, recurrent, and relapse cases and have been found to be helpful., ${ }^{5,10}$ Large lymph nodes that are symptomatic or lead to locoregional complications are amenable to surgical excision. Otherwise the disease tends to resolve spontaneously over several weeks to months. ${ }^{2,5,11}$

This case is unique for several reasons. First, the disease itself has a rare occurrence and very few patients present this late in life. Second, the unusual involvement of brachial plexus complicated the disease presentation, making it the second case ever reported in literature. Finally, the extensive involvement of lymph nodes that is, left supraclavicular, axillary, cervical, and superior mediastinal groups was also a striking feature.

\section{References}

1 Stelianides S, Belmatoug N, Fantin B. Manifestations and diagnosis of extrapulmonary tuberculosis [article in French]. Rev Mal Respir 1997;14(5, Suppl 5):S72-S87

2 Dorfman RF, Berry GJ. Kikuchi's histiocytic necrotizing lymphadenitis: an analysis of 108 cases with emphasis on differential diagnosis. Semin Diagn Pathol 1988;5(4): 329-345

3 Bosch X, Guilabert A, Miquel R, Campo E. Enigmatic KikuchiFujimoto disease: a comprehensive review. Am J Clin Pathol 2004;122(1):141-152

4 Wahid F, Rehman HU, Shahabi IK. Kikuchi's disease: a clinicopathological analysis of 22 cases. J Med Sci 2011;19(4): 177-180

5 Kucukardali Y, Solmazgul E, Kunter E, Oncul O, Yildirim S, Kaplan M. Kikuchi-Fujimoto disease: analysis of 244 cases. Clin Rheumatol 2007;26(1):50-54

6 Sudhakar MK, Sathyamurthy P, Indumathi E, et al. Kikuchi's disease: a case report from South India. Int J Case Rep Imag 2011; 2(2):15-18

7 Imamura M, Ueno H, Matsuura A, et al. An ultrastructural study of subacute necrotizing lymphadenitis. Am J Pathol 1982;107(3): 292-299

8 Kapoor S. Rare complications of Kikuchi's disease: beyond pain control. Korean J Pain 2012;25(4):281-282

9 Sugiyama A, Araki E, Arakawa K, et al. A case of subacute necrotizing lymphadenitis complicated with brachial plexus neuritis [article in Japanese]. Rinsho Shinkeigaku 1998;38(1011):941-944

10 Jang YJ, Park KH, Seok HJ. Management of Kikuchi's disease using glucocorticoid. J Laryngol Otol 2000;114(9):709-711

11 Sousa AdeA, Soares JM, Sá Santos MH, Martins MP, Salles JM. Kikuchi-Fujimoto disease: three case reports. Sao Paulo Med J 2010;128(4):232-235 